\title{
Adaptación del Inventario de Estrategias de Afrontamiento (CSI) a la Población Penitenciaria de Mexico
}

\author{
Adaptation of the Coping Strategies Inventory (CSI) \\ for the Mexican Prison Population
}

\author{
Francisco Javier Rodríguez-Díaz, ${ }^{*}$, Cristina Estrada-Pineda ${ }^{b}$ Luis Rodríguez-Franco $^{c}$ \\ $\&$ Carolina Bringas-Molleda ${ }^{d}$ \\ ${ }^{a}$ Universidad de Oviedo, Oviedo, Principado de Asturias, España,

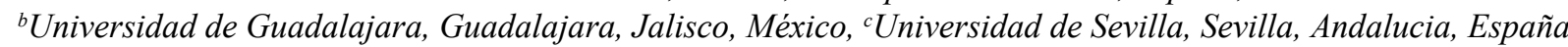 \\ \& ${ }^{d}$ Universidad de Valladolid, Valladolid, Provincia de Valladolid, España
}

\begin{abstract}
Resumen
El objetivo del estudio es adaptar el CSI - Inventario de Estrategias de Afrontamiento - al contexto penitenciario. La muestra - 261 penados, 97\% varones $(n=253)$ - del Sistema Postpenitenciario y Atención a Liberados (DSPAL) del Estado Jalisco, México. Los instrumentos utilizados: Ficha Penitenciaria de Historia de Vida y el Inventario CSI. Los resultados refieren una estructura de primer orden casi idéntica a la obtenida para la población general, con niveles de consistencia interna satisfactorios, al mismo tiempo que la interpretación de segundo orden no confirma la estructura de segundo y tercer orden. Se discuten las implicaciones de los resultados para intervención penitenciaria. Palabras clave: Estrategias de afrontamiento, adaptación, prisión, delito, conducta criminal.
\end{abstract}

\begin{abstract}
The aim of the present study is to adapt the Coping Strategies Inventory (CSI) to the prison context. The sample consisted of 261 prisoners, $97 \%$ were male $(n=253)$ from Sistema Postpenitenciario $y$ Atención a Liberados (DSPAL) of Jalisco, Mexico. The instruments used were Record of Prison Life History and the CSI. The results reported a first-order structure almost identical to that obtained for the general population with satisfactory levels of internal consistency whereas the results of second-order interpretation do not confirm the original structure of second and third order. The implications of the results for prison intervention are discussed.

Keywords: Coping strategies, adaptation, prison, crime, criminal behavior.
\end{abstract}

El proceso de afrontamiento es un aspecto central en la explicación de la adaptación de las personas a las dificultades de la vida cotidiana. Entendido como esfuerzos cognitivos y conductuales cambiantes, que se desarrollan para manejar las demandas específicas externas o internas (Lazarus \& Folkman, 1984), resultan un fructífero campo de investigación. La actualidad lo contempla desde variadas áreas de interés, donde destaca el análisis del efecto sobre la conducta y salud mental en diversas poblaciones,

\footnotetext{
* Dirección postal: Departamento de Psicología, Universidad de Oviedo, Campus de Oviedo, Plaza de Feijoo, s/n, Oviedo, Principado de Asturias, España 33003. E-mail: gallego@uniovi.es, cestraudg@hotmail.com, lurodri@ us.es y carolbringas@hotmail.com

Agradecimientos: Esta investigación fue financiada en su integridad por Fondos Mixtos de Fomento a la Investigación Científica y Tecnológica del Consejo Estatal de Ciencia y Tecnología del Estado de Jalisco (FOMIX COECYTJAL) y Gobierno del Estado de Jalisco, México (2008-08-98232) y Agencia Española de Cooperación Internacional para el Desarrollo - Programa Iberoamericano de Ciencia y Tecnología para el Desarrollo (AECID -CYTED) - AP/035718/11.
}

así como su utilidad en la adaptación a diversas condiciones vitales (Bouchard, 2003; Martínez Correa, Reyes, García León, \& González Jareño, 2006).

El afrontamiento ha sido y es un tema de interés, a la vez que productivo y controvertido. Productivo, por las repercusiones que la utilización de las estrategias de afrontamiento tienen en una amplia variedad de contextos clínicos, educativos, sociales, laborales, etc, e incluso dentro del ámbito penitenciario, para explicar el cambio hacia conductas normativas y evitación de comportamientos que favorecerían la reincidencia (L. A. Phillips \& Lindsay, 2011; Redondo, Martínez-Catena, \& Andrés-Pueyo, 2012; Reed, Alenazi, \& Potterton, 2009). Controvertido, en el sentido de que aún no ha sido establecida una estructura factorial básica acerca de los componentes fundamentales de las estrategias de afrontamiento y su consideración diferencial desde perspectivas teóricas muy diversas (Skinner, Edge, Altman, \& Sherwood, 2003).

Estas controversias se han trasladado a los sistemas de evaluación. En el idioma español, de acuerdo con Cano, Rodríguez y García (2007), no existen muchos 
instrumentos de evaluación adaptados; como refieren, la evaluación de las estrategias de afrontamiento (EA) en nuestro contexto presenta limitaciones que hacen necesario seguir trabajando en diseño, adaptación y refinamiento de la evaluación del afrontamiento. En esta línea, el estudio de Skinner et al. (2003) señala que dentro del amplio campo de instrumentos de evaluación disponibles en esta área, a nivel mundial, tan solo cuatro sistemas de categorización de EA han sido comprobados empíricamente.

Uno de los sistemas, que ha ofrecido resultados en esta área, ha sido el Coping Strategies Questionnaire (CSI), publicado por Tobin, Holroyd, Reynolds y Kigal (1989). Los autores han partido de una agrupación inicial de 109 ítems y tras aplicar un análisis factorial jerárquico se quedaron con 72 reactivos finales, que se han agrupan en ocho factores primarios, cuatro secundarios y dos terciarios. Adicionalmente, a diferencia de otros instrumentos de evaluación, requiere que el sujeto identifique previamente una situación de estrés sobre la cual responder al cuestionario.

La aplicación del CSI se ha realizado con éxito en el campo clínico, como es el caso de la discriminación de pacientes (depresivos, cefálgicos, neuróticos), indicándose especialmente su capacidad predictiva de síntomas depresivos en situación de elevado estrés (Tobin, 2001); otros ámbitos de aplicación son: problemas sociales (D'Zurilla \& Chang, 1995), bulimia nerviosa (Tobin \& Griffing, 1995), transplantados (Tix \& Frazier, 1998), enfermos de VIH (Bachanas et al., 2001), amputados (Livneh, Antonak, \& Gerhardt, 2000), estrés posttraumático (Najavits, Weiss, \& Shaw, 2000) entre otros. Al mismo tiempo, no conocemos estudios sobre el CSI en el ámbito penitenciario.

Desde esta realidad, se destaca que se dispone de la adaptación a muestras comunitarias del CSI al castellano, por Cano et al. (2007), obteniendo excelentes propiedades psicométricas que superaron incluso las del estudio original: ocho factores explicaron un $61 \%$ de la varianza con sólo 40 ítems (frente a los 72 que explicaban un $47 \%$ en el instrumento original), siendo los coeficientes de consistencia interna entre .63 y .89; sin embargo, no se ha confirmado la estructura de factores secundarios y terciarios. Este mismo estudio fue replicado en muestras latinoamericanas (Nava, Ollua, Vega, \& Soria, 2010), utilizando esta versión, que confirma la estructura factorial en los factores de primer orden, mientras la de segundo y tercer orden se asemejaron más a la estructura del estudio original (Tobin et al., 1989), al igual que en el caso de la versión abreviada de Addison et al. (2007), que utilizan una versión corta de dieciséis ítems del CSI (elaborada en el Jackson Heart Study, 2000), que ha confirmado la estructura factorial de segundo orden utilizada por el CSI, referido al manejo adecuado centrado en el problema vs manejo inadecuado centrado en el problema y manejo adecuado centrado en las emociones vs manejo inadecuado centrado en las emociones.

Los estudios previos, sin que se haya adaptado el instrumento al contexto penitenciario, de gran relevancia para enmarcar las EA en la subcultura carcelaria, demuestran estabilidad estructural del CSI en contextos muy variados.
Ello se refiere en tanto condición o factor predictivo, al modular el proceso de ayudar a las personas en que sean capaces de hacer frente a las situaciones a las que se enfrenta, y de forma autónoma. La aplicación de las EA en ambientes penitenciarios se realiza sobre la base de considerar, al menos, la prisión como un suceso traumático para una persona (S. D. Phillips, 2010; Rodríguez Díaz, Álvarez Fresno, García Zapico, Longoria, \& Noriega Carro, 2013; Rodríguez Díaz et al., 2011), que impacta y genera una cadena de estresores - dificultades de pareja, disminución de ingresos, comunicación con los seres queridos (hijos, padres, pareja, ...), alejamiento familiar, drogodependencia, ... (Degobbi \& Rezende, 2011; Mikulic \& Crespi, 2004; Moreno \& Mellizo, 2006), así como límites para manejar dificultades y generar cambios en la conducta (French \& Gendreau, 2006) y pensamientos (Giordano, Cernkovick, \& Rudolph, 2002), así como para mantenerlas estables a lo largo de la intervención con el interno para adaptarse a diferentes contextos (Blevins, Johnson, Cullen, \& Lero, 2010; Johnson, Colvin, Hanley, \& Flannery, 2010), dando lugar a nuevas pautas comportamentales, de roles y modalidades relacionales como forma de adaptarse a sus demandas y requerimientos (Pereira, Scheffer, \& Martins de Almeida, 2012; Rodríguez Díaz, Álvarez Fresno, García Zapico, Longoria, \& Noriega Carro, en prensa). Es decir, las EA son básicas a la hora de lograr construir una nueva historia de vida para el individuo que necesita de una nueva oportunidad, si cabe todavía más para aquellos que se refieren en el ámbito de la inadaptación social.

La necesidad descrita nos lleva a plantear como objetivo el adaptar el CSI al contexto penitenciario, verificando su estructura factorial en dicho ámbito penitenciario y el posible uso diferencial a realizar de las EA.

\section{Método}

\section{Participantes}

La muestra de estudio se determinó a través de un marco temporal, optándose por entrevistar a todas las personas que estuvieran asistiendo a la Dirección del Sistema Postpenitenciario y Atención a Liberados del Estado de Jalisco (DSPAL, en adelante), en el periodo de recuperación - entre los meses de Julio de 2009 a Febrero de 2010. Se excluyeron a los participantes que estuvieran integrados en los programas durante un periodo inferior a quince días de asistencia, así como aquellos con algún problema psiquiátrico puesto de manifiesto previamente por la historia penitenciaria y/o en estado de embriaguez o efectos de alguna sustancia psicoactiva, en el momento de la entrevista, detectado por el entrevistador tanto por sintomatología física como en las propias relaciones interpersonales.

La muestra final quedó conformada por 261 liberados privados de libertad, de los que el $97 \%$ eran varones $(n=253)$ que cumplían condena en el Postpenitenciario de Jalisco (México). El delito más relevante fue el robo calificado $(n=228)$ con un $87,40 \%$, refiriendo una frecuente actividad delictiva previa detectada desde edades 
Rodríguez-Díaz, J. F., Estrada-Pineda, C., Rodríguez-Franco, L. \& Bringas-Molleda, C. Adaptación del Inventario de Estrategias de Afrontamiento (CSI) a la Población Penitenciaria de Mexico.

tempranas en más del 50\%, que llega a ser reconocida con anterioridad a los 12 años - primera detención previa a los 18 años en el $21 \%(n=55)$. El rango de edad de los participantes se ha situado entre los 20 y 61 años, siendo el estado civil predominante el de soltero $(n=115)$ con un 44,10\% de la muestra - Unión Libre $(n=77 ; 29,50 \%)$, casado ( $n=49 ; 18,80 \%)$-, mientras el $44 \%(n=114)$ vivía con la familia origen y la convivencia con esposa o pareja era del 20\% $(n=53)$. El nivel de escolarización de los participantes fue bajo (más del $50 \%$ no han completado estudios de secundaria), sin reconocer problemática escolar a lo largo del periodo de escolarización - cerca del 50\%.

Se autoadscriben a un nivel socioeconómico de clase media ( $n=201$ ) en un $77 \%$, mientras refieren encontrarse desempleados el 15,30\% $(n=40)$ y desarrollan una actividad en la economía informal el $21,10 \%(n=55)$. A la vez, el ámbito familiar de origen no refiere la presencia destacada de antecedentes penales $(n=226)$ en un $86 \%$, aunque se detecta en más de un $70 \%(n=183)$ el consumo de sustancias psicoactivas, mientras que el propio consumo supera el $90 \%(n=242)$. Por el contrario, la problemática de Salud Mental es baja, al no superar el 8\% $(n=20)$.

\section{Instrumentos}

La batería de evaluación empleada comprendía un cuestionario ad hoc a manera de entrevista semiestructurada - Ficha Penitenciaria de Historia de Vida (Estrada, Rodríguez, \& Solano, 2012), que ha buscado establecer las características sociodemográficas y el proceso de socialización (familiar, escolar y laboral) como la historia de vida de integración comunitaria (adicciones, penitenciaria, ...) de los liberados participantes.

Adicionalmente, se utilizó el inventario de Tobin et al. (1989), para evaluar las EA, en su adaptación de 40 ítems de Cano et al. (2007). En ésta, el liberado debe transcribir, en primer lugar, una situación estresante relacionada con su periodo penitenciario para contestar con posterioridad, a través de una escala likert, lo referido a la utilización de EA. Estas situaciones han sido agrupadas posteriormente en cinco categorías: Problemas de reclusión y derivados (situaciones del interno en reclusión y/o aquellas por estar en tal situación); Desadaptación Social (problemas de adaptación a integrarse en contextos de barrio y laborales); Problemas familiares (problemas de la familia de origen y con su pareja); Problemas de relaciones interpersonales con amigos, víctimas, coetáneos o personas relativas al apoyo formal y otros (refieren problemas con adicciones, remordimiento por el delito, salud, ...). Las respuestas a los ítems se han recogido a través de una escala tipo Likert de cinco puntos, que define la frecuencia de lo que hizo en la situación descrita, identificando sus respuestas ocho estrategias primarias, cuatro secundarias y dos terciarias.

Las ocho escalas primarias son: Resolución de problemas, Reestructuración cognitiva, Apoyo social, Expresión emocional, Evitación de problemas, Pensamiento desiderativo, Retirada social, Autocrítica. Las escalas secundarias surgen de la agrupación empírica de las primarias, es decir, Manejo adecuado centrado en el problema - incluye
Resolución de problemas y Reestructuración cognitiva, indicando un afrontamiento adaptativo centrado en el problema, bien modificando la situación, bien su significado-, Manejo adecuado centrado en la emoción - subescalas Apoyo social y Expresión emocional, reflejando un afrontamiento adaptativo centrado en el manejo de las emociones que afloran en el proceso estresante-, Manejo inadecuado centrado en el problema - subescalas Evitación de problemas y Pensamiento desiderativo, indicando un afrontamiento desadaptativo centrado en el problema, bien evitando las situaciones estresantes, bien fantaseando sobre realidades alternativas pasadas, presentes o futuras - y Manejo inadecuado centrado en la emoción- subescalas Retirada social y Autocrítica, reflejando un afrontamiento desadaptativo centrado en las emociones, pero basado en el aislamiento, la autocrítica y la autoinculpación. Las escalas terciarias surgen de la agrupación empírica de las secundarias, es decir, Manejo adecuado: subescalas de Resolución de problemas, Reestructuración cognitiva, Apoyo social y Expresión emocional, indicando esfuerzos activos y adaptativos por compensar la situación estresante; Manejo inadecuado: subescalas Evitación de problemas, Pensamiento desiderativo, Retirada social y Autocrítica, sugiriendo afrontamiento pasivo y desadaptativo.

Para finalizar, se completó con un ítem adicional sobre la autoeficacia percibida (¿En qué grado manejó adecuadamente la situación?) con un sistema de respuesta de: nada, algo, bastante, mucho, totalmente

\section{Procedimiento}

La recogida de datos ha contado con la colaboración y apoyo de los directivos de la DSPAL, institución que registra y supervisa la reinserción de liberados a la comunidad, la cual ha ofrecido todas las facilidades al equipo de investigadores para realizar las actividades necesarias. La aplicación se hizo en el tiempo que tienen asignado los liberados para su trabajo comunitario, aunque tratando de no impactar en actividades consideradas importantes por el sistema para su reinserción. Al aceptar, se entregaba para su lectura una carta de consentimiento informado, debiendo ellos expresar verbalmente su aceptación. Aceptada su participación, se realizaba la entrevista - el flujo de entrevistados a las instalaciones de la DSPAL fue lento, por lo que las 261 entrevistas han exigido mantener la evaluación durante siete meses.

El análisis de datos, utilizando el paquete estadístico SPSS 18.0, ha buscado identificar la estructura interna e índice de consistencia del inventario, recurriendo para ello al análisis factorial y el índice Alpha de Cronbach. Para cada uno de los factores resultantes se han obtenido las puntuaciones directas, al igual que han sido agrupadas las diferentes situaciones de estrés sobre las cuales los liberados han identificado sus estrategias de afrontamiento utilizadas y el grado de eficacia alcanzado, incluyendo los procedimientos el análisis correlacional entre los factores y entre estos y la percepción de eficacia, a la vez que el análisis de varianza (ANOVA) para determinar su utilización frente a las diferentes realidades de estrés percibidas. 


\section{Resultados}

Se realiza un análisis factorial exploratorio (componentes principales, autovalores iguales o superiores a uno, rotación ortogonal), obteniéndose un total de 11 factores, que explican el $60 \%$ de varianza total (ver Tabla 1) - oscilan entre el 8 y el 3\%. Los primeros siete factores son una réplica de los hallados en el estudio original (Tobin et al., 1989) y en otros dos posteriores (Cano et al., 2007; Nava et al., 2010) - ver Tabla 2.

El primer factor refiere la expresión emocional, donde en relación al estudio original se advierte la no inclusión del ítem 35 . El segundo factor corresponde al factor de autocrítica, que reproduce el obtenido en el estudio original. El tercer factor, manteniendo la estructura de la propuesta original, se adscribe como retirada social. El factor de re- solución de problemas sigue la misma tónica anterior, lo mismo que sucede con el quinto factor (reestructuración cognitiva). Los ítems 15, 23, 31 y 39 componen el factor de evitación de problemas, observándose en relación con la propuesta original la ausencia del ítem número 7; algo similar sucede con el factor de pensamiento desiderativo (ítems 4, 12, 20 y 28), que no incluye el ítem 36.

Por último, el factor original apoyo social ha aparecido dividido en dos factores (números 8 y 9 de la Tabla 1): el primero conformado por ítems 13,5 y 37 y el segundo 21 y 29. El análisis de consistencia interna de estos dos factores ofrece un índice Alpha de Cronbach de .76 y de .28 respectivamente. Es precisamente este último valor de consistencia interna tan bajo el que nos ha llevado a optar a la consideración conjunta de ambos factores, obteniéndose un índice alpha de .75 .

Tabla 1

Matriz de Componentes Rotados del CSI con el Método de Extracción de Análisis de Componentes Principales (Método de rotación: Normalización Varimax con Kaiser)

\begin{tabular}{rlllllll}
\hline & EE AU RS RP & RC EP PD
\end{tabular}

27. Dejé desahogar mis emociones

19. Analice mis sentimientos y simplemente los dejé salir

3. Dejé salir mis sentimientos para reducir el estrés

11. Expresé mis emociones, lo que sentía

26. Me recriminé por permitir que esto ocurriera

10. Me di cuenta que era personalmente responsable de mis dificultades y me lo reproché

34. Fue un error mío, así que tenía que sufrir las consecuencias

2. Me culpé a mí mismo

18. Me critiqué por lo ocurrido

8. Pasé algún tiempo solo .753

16. Estar con gente .668

24. Oculté lo que pensaba y sentía .625

40. Traté de ocultar mis sentimientos

32. No dejé que nadie supiera como me sentía $-.397$ .531

8. Me esforcé para resolver los problemas de la situación

17. Hice frente al problema

33. Mantuve mi postura y luche por lo que quería

25. Supe lo que había que hacer, así que doble mis esfuerzos 
Rodríguez-Díaz, J. F., Estrada-Pineda, C., Rodríguez-Franco, L. \& Bringas-Molleda, C. Adaptación del Inventario de Estrategias de Afrontamiento (CSI) a la Población Penitenciaria de Mexico.

22. Me convencí de que las cosas no eran tan malas como parecían

30. Me pregunté qué era realmente importante y descubrí que las cosas no estaban tan mal después de todo

14. Cambié la forma en que veía la situación para que las cosas no parecieran malas

38. Me fijé en el lado bueno de las cosas

6. Repasé el problema una y otra vez en mi mente y al final vi las cosas de una forma diferente

31. Me comporté como si nada hubiera pasado

23. Quité importancia a la situación y no quise preocuparme más

20. Deseé no encontrarme nunca más en esa situación

28. Deseé poder cambiar lo que había sucedido

4. Deseé que la situación nunca hubiera empezado

12. Deseé que la situación no existiera o que de alguna manera terminase

Nota. EE: Expresión Emocional; AU: Autocrítica; RS: Retirada Social; RP: Resolución de Problemas; RC: Reestructuración Cognitiva; EP: Evitación de Problemas; PD: Pensamiento Desiderativo.

Tabla 2

Comparativa de Datos Psicométricos del CSI obtenidos en el Estudio Original y en Otros dos Posteriores Diferentes Estudios Realizados con Población Hispanoamericana

\begin{tabular}{|c|c|c|c|c|c|c|c|c|c|c|c|c|c|}
\hline & \multicolumn{3}{|c|}{ Estudio Actual } & \multicolumn{3}{|c|}{$\begin{array}{l}\text { Tobin et al. } \\
\text { (1989) }\end{array}$} & \multicolumn{3}{|c|}{$\begin{array}{l}\text { Cano et al. } \\
(2007)\end{array}$} & \multicolumn{3}{|c|}{$\begin{array}{l}\text { Nava et al. } \\
(2010)\end{array}$} & \multirow[t]{2}{*}{ Ejemplo } \\
\hline & Ítems & Var. & $\mathrm{a}$ & Ítems & Var. & a & Ítems & Var. & a & Ítems & Var. & A & \\
\hline AUT & 5 & $7,4 \%$ & .80 & 9 & $12 \%$ & .94 & 5 & $9 \%$ & .89 & 5 & $9 \%$ & .86 & $\begin{array}{l}\text { Me recriminé por permitir que esto } \\
\text { ocurriera }\end{array}$ \\
\hline RES & 5 & $6,6 \%$ & .80 & 9 & $4 \%$ & .81 & 5 & $5 \%$ & .65 & 5 & $6 \%$ & .78 & Pasé algún tiempo solo \\
\hline REP & 5 & $6,2 \%$ & .73 & 9 & $5 \%$ & .82 & 5 & $9 \%$ & .86 & 5 & $8 \%$ & .80 & Luché para resolver el problema \\
\hline REC & 5 & $6,2 \%$ & .69 & 9 & $3 \%$ & .83 & 5 & $7 \%$ & .80 & 5 & $7 \%$ & .82 & $\begin{array}{l}\text { Me convencí de que las cosas no } \\
\text { eran tan malas como parecían }\end{array}$ \\
\hline APS & 5 & $9,4 \%$ & .75 & 9 & $6 \%$ & .89 & 5 & $8 \%$ & .80 & 5 & $8 \%$ & .84 & $\begin{array}{l}\text { Hablé con una persona de } \\
\text { confianza }\end{array}$ \\
\hline EEM & 4 & $8 \%$ & .79 & 9 & $6 \%$ & .89 & 5 & $8 \%$ & .84 & 5 & $8 \%$ & .86 & Dejé desahogar mis emociones \\
\hline EVP & 4 & $5,4 \%$ & .62 & 9 & $7 \%$ & .72 & 5 & $6 \%$ & .63 & 5 & $7 \%$ & .70 & $\begin{array}{l}\text { Me comporté como si nada hubiera } \\
\text { pasado }\end{array}$ \\
\hline PSD & 4 & $5,1 \%$ & .62 & 9 & $4 \%$ & .78 & 5 & $8 \%$ & .78 & 5 & $8 \%$ & .84 & $\begin{array}{l}\text { Deseé no encontrarme nunca más } \\
\text { en esa situación }\end{array}$ \\
\hline Total & 37 & $54,23 \%$ & & 72 & $47 \%$ & & 40 & $60 \%$ & & 40 & $62 \%$ & & \\
\hline
\end{tabular}

Nota. EEM: Expresión Emocional; AUT: Autocrítica; RES: Retirada Social; REP: Resolución de Problemas; REC: Reestructuración cognitiva; EVP: evitación de problemas; PSD: pensamiento desiderativo; APS: apoyo social. 
Una vez replicada la estructura factorial de primer orden, procedimos a comprobar la estructura de factores de segundo orden del CSI. Para ello se realizó un análisis factorial partiendo de las puntuaciones obtenidas en los ocho factores primarios, utilizando el mismo sistema de extracción que previamente se había utilizado, obteniendo una solución trifactorial (Tabla 3) con varianzas explicadas entre el $32 \%$ y el $15 \%$, siendo el total del $66 \%$.

Tabla 3

Matriz de Componentes Rotados Resultantes del Análisis Factorial de Segundo Orden (saturaciones superiores a .50) Mediante el Método de Extracción de Componentes Principales - Método de Rotación: Normalización Varimax con Kaiser

\begin{tabular}{lccc}
\hline \multicolumn{4}{c}{ Matriz de componentes rotados } \\
& \multicolumn{3}{c}{ Componente } \\
\cline { 2 - 4 } & 1 & 2 & 3 \\
\hline Apoyo social (AS) & .828 & \\
Expresion emocional (EE) & .773 & \\
Retirada social (RS) & -.708 & \\
Resolución de problemas (RP) & .612 & \\
Reestructuración cognitiva (RC) & .610 & .792 \\
Autocrítica (AU) & & .733 \\
Pensamiento desiderativo (PD) & & \\
Evitación de problemas (EP) & & .910 \\
\hline
\end{tabular}

Nota. La rotación ha convergido en 5 iteraciones. 1. Manejo adecuado de problemas; 2. Manejo emocionalmente inadecuado de problemas; 3. Manejo inadecuado de problemas.

El primer factor obtenido nos muestra una combinación, en relación al estudio original, de estrategias relacionadas con el manejo adecuado centrado en la emoción (AS y EE), de manejo adecuado centrado en la resolución de problemas (RP y RC) y de manejo inadecuado centrado en la emoción (RS, con carga negativa). En suma, parece corresponder al factor original terciario de manejo adecuado (sea centrado en el problema o en la emoción), aunque incluye un factor adicional (Retirada Social) que parece que en este contexto no puede descartarse como eficaz. Por tanto, no comprobamos en nuestra muestra la estructura factorial de órdenes superiores del CSI, aunque los factores hallados parecen estar orientados a los niveles de eficacia de las estrategias de afrontamiento utilizadas, donde el segundo factor (conformado por autocrítica y pensamiento desiderativo) incluye dimensiones que originariamente se encontraban adscritas a los factores secundarios de manejo inadecuado emocional y basado en problemas, respectivamente.

La matriz de intercorrelaciones entre las estrategias de afrontamiento - ver Tabla 4, arrojó una proporción muy elevada de índices de correlación estadísticamente significativos entre los distintos factores. La resolución del problemas apareció como la estrategia de afrontamiento con mayor número de correlaciones estadísticamente significativas con las restantes escalas del cuestionario, siendo negativas con retirada social y evitación de problemas; la escala de reestructuración cognitiva, a su vez, presentó correlaciones estadísticamente significativas con el conjunto de las escalas a excepción del pensamiento desiderativo, siendo negativas con la retirada social; la expresión emocional presenta su vez correlaciones estadísticamente significativas con el conjunto de las restantes relaciones a excepción de la evitación de problemas. Es de resaltar las correlaciones no significativas del apoyo social con respecto a autocrítica y Pensamiento desiderativo.

$\mathrm{El}$ análisis de la eficacia percibida de las estrategias de afrontamiento - ver Tabla 5 - muestra correlaciones positivas significativas con 4 factores (Resolución de Problemas, Reestructuración Cognitiva, Expresión Emocional, Apoyo Social), más uno adicional con valores negativos (Retirada Social).

Finalmente, se codificaron los tipos de situaciones que los internos habían señalado como especialmente estresantes en su medio penitenciario - ver Tabla 6. Aunque destacan especialmente la proporción de problemas propios de la reclusión y los familiares (señalados en su conjunto por los propios internos como problemas destacados en más del $66 \%$ de los casos), la casuística es variada, siendo ello relevante a considerar que estas categorías identifican el objetivo sobre el que se utilizan las estrategias de afrontamiento de los internos. Ante ello, se procedió a realizar un análisis de varianza sobre las puntuaciones obtenidas en los factores primarios obtenidos en nuestro estudio en función del tipo de situaciones planteadas por los internos, apareciendo valores cercanos a la significación para el apoyo social $(F=2,375 ; p=.053)$ y evitación de problemas $(F=2,243 ; p=.065)$. 
Rodríguez-Díaz, J. F., Estrada-Pineda, C., Rodríguez-Franco, L. \& Bringas-Molleda, C. Adaptación del Inventario de Estrategias de Afrontamiento (CSI) a la Población Penitenciaria de Mexico.

Tabla 4

Matriz de Intercorrelaciones entre las Estrategias de Afrontamiento del Cuestionario CSI y Nivel de Significación de Éstas

\begin{tabular}{|c|c|c|c|c|c|c|c|}
\hline & AU & $\mathrm{RS}$ & $\mathrm{RP}$ & $\mathrm{RC}$ & EP & PD & AS \\
\hline $\mathrm{EE}$ & $.167 * *$ & $-.409 * *$ & $.382 * *$ & $.380 * *$ & -.056 & $.162 * *$ & $.558 * *$ \\
\hline AU & & $.162 * *$ & $.262 * *$ & $.281 * *$ & -.026 & $.293 * *$ & .091 \\
\hline RS & & & $-.246^{* *}$ & $-.158 *$ & $.278 * *$ & $.134^{*}$ & $-.526^{* *}$ \\
\hline $\mathrm{RP}$ & & & & $.406 * *$ & $-.124^{*}$ & $.188^{*}$ & $.394 * *$ \\
\hline $\mathrm{RC}$ & & & & & $.163 * *$ & .087 & $.374 * *$ \\
\hline EP & & & & & & .042 & $-.143 *$ \\
\hline PD & & & & & & & .095 \\
\hline
\end{tabular}

Nota. EE: Expresión Emocional; AU: Autocrítica; RS: Retirada Social; RP: Resolución de Problemas; RC: Reestructuración cognitiva; EP: evitación de problemas; PD: pensamiento desiderativo; AS: apoyo social

$* p<.05 ; * * p<.01$

Tabla 5

Estrategias de Afrontamiento y Eficacia Percibida por los Internos en la Resolución de Situaciones Estresantes

\begin{tabular}{lcccccccc}
\hline & EE & AU & RS & RP & RC & EP & PD & AS \\
\hline EFICACIA & $.191 * *$ & .028 & $-.156^{*}$ & $.360^{* *}$ & $.153^{*}$ & -.074 & .089 & $.141^{*}$ \\
\hline
\end{tabular}

Nota. EE: Expresión Emocional; AU: Autocrítica; RS: Retirada Social; RP: Resolución de Problemas; RC: Reestructuración cognitiva; EP: evitación de problemas; PD: pensamiento desiderativo; AS: apoyo social

$* p<.05 ; * * p<.01$.

Tabla 6

Tipo de Situaciones Afrontadas por los Internos Señaladas como Especialmente Estresantes en el Medio Penitenciario

\begin{tabular}{lcc}
\hline & Frecuencia & Porcentaje \\
\hline Problemas de la reclusión y derivados & 84 & 32,2 \\
Desadaptación social en integración & 48 & 18,4 \\
Problemas familiares & 89 & 34,1 \\
Problemas interpersonales & 28 & 10,7 \\
Otros & 12 & 4,6 \\
\hline Total & 261 & 100,0 \\
\hline
\end{tabular}

\section{Discusión}

Aunque publicaciones de adaptaciones españolas previas sugieren que el instrumento ha mostrado en poblaciones clínicas una estructura de primer orden estable (no así en la propuesta de segundo y tercer orden) y unos niveles de consistencia interna adecuados, el CSI no ha sido adaptado a otros contextos donde la aparición de distintos estresores puedan favorecer y explicar la presencia de diversas estrategias de adaptación. Entre ellos se encontraría el contexto penitenciario.
El objetivo del trabajo era adaptar al medio penitenciario de nuestro entorno el CSI, comprobando sus propiedades psicométricas en una muestra distinta a la población general, así como interpretar la resolución de situaciones estresantes con personas privadas de libertad. Los resultados refieren que el instrumento ofrece una estructura de primer orden casi idéntica a aquella obtenida para la población general (Cano et al., 2007; Nava et al., 2010; Tobin et al., 1989), con la excepción del factor de apoyo social - en nuestro caso se ha distribuido en dos factores, que se explica desde el contexto donde el sujeto 
vive y percibe los estresores, es decir, el primero de ellos más centrado en el ámbito de privación de libertad, mientras el segundo de ellos se relaciona con la vivencia en la situación de libertad.

El CSI ofrece niveles de consistencia interna satisfactorios - entre .63 y .80-, en la línea de los resultados de Cano et al. (2007) y Nava et al. (2010), al mismo tiempo que la interpretación resultante del análisis factorial de segundo orden a través de la matriz rotada no confirma la estructura original de segundo y tercer orden del cuestionario. Como se aprecia en la Tabla 3, básicamente aparecen dos factores de segundo orden. El primero incluye factores primarios de muy diversa naturaleza que, siguiendo la denominación original de Tobin y sus colaboradores, incluye estrategias adaptativas (sean centradas en el problema o en las emociones), más un factor adicional con carga negativa que en el estudio original aparecía como estrategia desadaptativa. Por tanto, y aunque no se confirme la estructura del cuestionario, podríamos denominar a este primer factor como de afrontamiento adaptativo. En el segundo de los factores de segundo orden hallados en nuestro estudio, saturan dos factores primario que originariamente eran denominados como desadaptativos, aunque en nuestro caso incluyen estrategias emocionales y conductuales. En suma, la estructura de segundo orden de nuestro estudio en población penitenciaria parece asemejarse más a la terciaria hallada por el equipo de Tobin en su estudio original.

Por otro lado, como se detalla en la Tabla 4, los factores primarios se encuentran correlacionados entre sí, lo que corrobora estudios anteriores. Ello hay que interpretarlo en el sentido de que las estrategias de afrontamiento muestran un patrón complejo de interacciones y probablemente habitualmente sean utilizadas de forma conjunta y simultánea (algunas con mayor frecuencia que otras) en los intentos de solución de problemas dependiendo del contexto penitenciario (subcultura carcelaria vs no).

Este dato resulta relevante al considerar el efecto que el uso de las EA pueda repercutir sobre la eficacia del afrontamiento. Los resultados muestran el elenco de estrategias consideradas por los internos como eficaces o no y ello se puede utilizar como ayuda para el desarrollo de programas de mejora de las habilidades de los propios internos en el medio penitenciario en vistas a su adaptación posterior en el contexto vital. Sin embargo, desafortunadamente, no hemos encontrado estudios que analicen el efecto del uso combinado de las EA, ya que habitualmente su eficacia ha sido comprobada de forma aislada. Por ello, se necesitan realizar estudios que marquen las relaciones entre la eficacia percibida de los internos con criterios externos y objetivos de eficacia -la medida de eficacia percibida debe tomarse solo como un indicador de la percepción de los internos acerca del uso de sus estrategias de afrontamiento y no como un indicador contrastado ni objetivo una manera de afrontar eficientemente un problema, sin olvidar que estudios previos han considerado diversas variables (tanto personales como situacionales) que modulan el efecto y, por tanto, la eficacia de las EA, lo que hace complejo el análisis de la eficacia de las EA en nuestro estudio (Carver \& Connor-Smith, 2010). Estas consideraciones han de analizarse en próximos estudios.

Otro dato a señalar especialmente son las situaciones sobre las que han sido evaluadas las diferentes EA, en las que hemos detectado una problemática muy diversa - predominan los problemas familiares que han surgido durante la reclusión (en un 34\% de los casos elegida como situación estresante por los internos) y los problemas directos derivados de la misma reclusión (en un 32\%). Los datos preliminares de nuestro estudio señalan que el uso de las estrategias de afrontamiento han sido realizadas casi con independencia del tipo de situación problemática; además, los propios participantes señalan el uso de EA que han sido consideradas por ellos mismos como ineficaces (la retirada social y la evitación de problemas) lo que plantea la posibilidad de establecer perfiles diferenciales explicativos y con relación a la reincidencia en la carrera delictiva.

En cualquier caso, la complejidad del problema que plantea estas cuestiones, nos hace derivar a un estudio posterior su planteamiento más pormenorizado donde responder respecto a las dimensiones de estrategias de afrontamiento y a la percepción de eficacia de éstas por sujetos privados de libertad, refiriendo el afrontamiento tanto como foco u orientación del mismo - aproximación al problema vs evitación- como por método implementado - cognitivo vs conductual. Se confirma la presencia, en el contexto penitenciario, de diversas formas de respuesta frente a situaciones percibidas como estresantes, es decir, focalizadas en el problema con expresión cognitiva y conductual, con bases resultantes a destacar en el apoyo social para manejar la realidad, y aquellas centradas en la retirada como en la autoinculpación y/o refiriendo intentos cognitivos de no pensar en el estresor y sus implicaciones, sin contar con apoyo social, es decir, en base a su aislamiento y sin expresión emocional.

Nuestro estudio, además de adaptar el CSI al contexto penitenciario, es un primer paso para evaluar tanto el poder explicativo del uso de determinadas estrategias de afrontamiento en la población penada a la hora de afrontar su vida en libertad y su incidencia en la reincidencias delictiva, como a que estrategias dan prioridad los penados ante un determinado estresor en una determinada realidad percibida como estresora. Los resultados, a su vez, nos llevan a plantear en futuras investigaciones el analizar el tipo de estrategias que priman en los internos penitenciarios de acuerdo con el grado de desajuste, determinando la influencia tanto en la propia situación de reclusión como en el grado de adaptación futura a la vida en libertad, desde el criterio de reincidencia delictiva, pero no únicamente desde la perspectiva de ausencia de delito, ya que ello podría incluso justificar una buena adaptación a la subcultura carcelaria. 
Rodríguez-Díaz, J. F., Estrada-Pineda, C., Rodríguez-Franco, L. \& Bringas-Molleda, C. Adaptación del Inventario de Estrategias de Afrontamiento (CSI) a la Población Penitenciaria de Mexico.

\section{Referencias}

Addison, C. C., Campbell-Jenkins, B. W., Sarpong, D. F., Kibler, J., Singh, M., Dubbert, P., ... Taylor, H. (2007). Psychometric evaluation of a Coping Strategies Inventory Short-Form (CSI$\mathrm{SF}$ ) in the Jackson Heart Study Cohort. International Journal of Environmental Research Public Health, 4(4), 289-295.

Bachanas, P. J., Kullgren, K. A., Schwartz, K. S., Lanier, B., McDaniel, J. S., Smith, J., ...Nesheim, S. (2001). Predictors of psychological adjustment in school-age children infected with HIV. Journal of Pediatric Psychology, 26, 343-352.

Blevins, K. R., Johnson, S., Cullen, F. T., \& Lero, C. (2010). A general strain Theory of Prison Violence and Misconduct: An integrated model of inmate behavior. Journal of Contemporary Criminal Justice, 26(2), 148-166.

Bouchard, G. (2003). Cognitive appraisals, neuroticism and openness as correlates of coping strategies: An integrative model of adaptation to marital difficulties. Canadian Journal of Behaviour Science, 35, 1-12.

Cano, F. J., Rodríguez Franco, L., \& García Martínez, J. (2007). Adaptación española del Inventario de Estrategias de Afrontamiento. Actas Españolas de Psiquiatría, 35, 29-39.

Carver, C. S., \& Connor-Smith, J. (2010). Personality and coping. Annual Review of Psychology, 61, 679-704.

Degobbi, L., \& Rezondo, M. (2011). Experiências infantis e risco de abuso físico: Mecanismos envolvidos na repetição da violência. Psicologia: Reflexão e Crítica, 24(4), 710-719.

D'Zurilla, T. J., \& Chang, E. C. (1995). The relations between social problem solving and coping. Cognitive Therapy and Research, 19(5), 547-562.

Estrada, C., Rodríguez, F. J., \& Solano, C. (2012). Castigo y carrera delictiva de liberados del Sistema Penitenciario de Jalisco. In S. P. Colín Soto \& E. García-López (Eds.), Más allá de la violencia. Retos hacia la reconstrucción (pp. 291-320). Morelia, Mexico: Universidad de Monterrey.

French, S. A., \& Gendreau, P. (2006). Reducing prison misconduct: What works? Criminal Justice and Behavior, 33, 185-218.

Giordano, P. C., Cernkovich, S. A., \& Rudolph, J. L. (2002). Gender, crime, and desistance: Towards a theory of cognitive transformation. American Journal of Sociology, 107, 990-1064.

Johnson, S., Colvin, M., Hanley, D., \& Flannery, D. (2010). Victimization, social support, and psychological well-being: A study of recently released prisoners. Criminal Justice and Behavior, 37, 1140-1159.

Lazarus, R. S., \& Folkman, S. (1984). Stress, appraisal and coping. New York: Springer.

Livneh, H., Antonak, R. F., \& Gerhardt, J. (2000). Multidimensional investigation of the structure of coping among people with amputations. Psychosomatics, 41, 235-244.

Martínez Correa, A., Reyes, G., García León, A., \& González Jareño, M. I. (2006). Optimismo/pesimismo disposicional y estrategias de afrontamiento del estrés. Psicothema, 18, 66-72.

Mikulic, I. M., \& Crespi, M. (2004). Contexto carcelario: un estudio de los estresores y las respuestas de afrontamiento en detenidos primarios y reincidentes. In XII Anuario de Investigaciones (Vol. 2, pp. 211-221). Buenos Aires, Argentina: Facultad de Psicología, Universidad de Buenos Aires.

Moreno, C. A., \& Mellizo, L. F. (2006, abril). Familias de internos e internas de cárcel: una revisión de la literatura. Documento presentado en el III Congreso Latinoamericano Virtual de Psicología Jurídica y Forense, Bogotá, Colombia.
Najavits, L. M., Weiss, R. D., \& Shaw, S. R. (2000). A clinical profile of women with PTSD and substance dependence. Psychology Addictive Behaviors, 13, 98-104.

Nava, C., Ollua, P., Vega, C. Z., \& Soria, R. (2010). Inventario de Estrategias de Afrontamiento: una replicación. Psicología y Salud, 20(2), 213-220.

Pereira, G., Scheffer, M., \& Martins de Almeida, R. M. (2012). Drogas, violência e aspectos emocionais em apenados. Psicologia: Reflexão e Crítica, 25(1), 89-95.

Phillips, S. D. (2010). The past as prologue: Parental incarceration, service planning, and intervention development in context. In J. M. Eddy \& J. Poehlmann (Eds.), Children of incarcerated parents: A handbook for researchers and practitioners (pp. 13-32). Washington, DC: The Urban Institute Press.

Phillips, L. A., \& Lindsay, M. (2011). Prison to society: A mixed methods analysis of coping with reentry. International Journal of Offender Therapy and Comparative Criminology, 55(1), 136-154.

Redondo, S., Martínez-Catena, A., \& Andres-Pueyo, A. (2012). Therapeutic effects of a cognitive-behavioural treatment with juvenile offenders. The European Journal of Psychology Applied to Legal Context, 4(2), 159-178.

Reed, P., Alenazi, Y., \& Potterton, F. (2009). Effect of time in prison on prisoners' use of coping strategies. International Journal of Prisoner Health, 5(1), 16-24.

Rodríguez Díaz, F. J., Álvarez Fresno, E., García Zapico, F., Longoria, B., \& Noriega, M. I. (2013). El sistema penitenciario: un análisis desde el ayer para el mañana. In E. García-López (Ed.), Psicopatología forense. Comportamiento humano y tribunales de justicia. Assunción, Paraguay: Manual Moderno.

Rodríguez Díaz, F. J., Álvarez Fresno, E., García Zapico, F., Longoria, B., \& Noriega, M. I. (en prensa). Modelo alternativo de intervención educativa en el contexto penitenciario. Propuesta desde la UTE de Villabona (España). Revista Aula Abierta.

Rodríguez Díaz, F. J., Bringas, C., Rodríguez Franco, L., LópezCepero, J., Pérez, B., \& Estrada, C. (2011). Drug abuse and criminal family records in the criminal history of prisoners. The European Journal of Psychology Applied to Legal Context, 3(2), 89-106.

Skinner, E. A., Edge, K., Altman, J., \& Sherwood, H. (2003). Searching for the structure of coping: A review and critique of category systems for classifying ways of coping psychological. Bulletin Copyright, 129(2), 216-269.

Tix, A. P., \& Frazier, P. A. (1998). The use of religious coping during stressful life events. Journal of Consulting and Clinical Psychology, 66, 411-422.

Tobin, D. L. (2001). User manual for the Coping Strategies Questionnaire. Retrieved June 15, 2012, from http://www. ohioupsychology.com/files/Manual\%20Coping\%20Strategies\%20Inventory.pdf

Tobin, D. L., \& Griffing, A. S. (1995). Coping and depression in bulimia nervosa. International Journal of Eating Disorders, 18(4), 359-363.

Tobin, D. L., Holroyd, K. A., Reynolds, R. V., \& Kigal, J. K. (1989). The hierarchical factor structure of the Coping Strategies Inventory. Cognitive Therapy Resesearch, 13, 343-361.

Recebido: 08/01/2013

$1^{a}$ revisão: $13 / 03 / 2013$

Aceite final: 19/03/2013 